\title{
Quantum Nucleation in a Ferromagnetic Film Placed in a Magnetic Field at an Arbitrary Angle
}

\author{
Gwang-Hee Kim* \\ Department of Physics, Sejong University, Seoul 143-747, Korea \\ (Received \\ )
}

\begin{abstract}
We study the quantum nucleation in a thin ferromagnetic film placed in a magnetic field at an arbitrary angle. The dependence of the quantum nucleation and the temperature of the crossover from thermal to quantum regime on the direction and the strength of the applied field are presented. It is found that the maximal value of the rate and that of the crossover temperature are obtained at a some angle with the magnetic field, not in the direction of the applied field opposite to the initial easy axis.
\end{abstract}

PACS numbers: 75.45.+j, 73.40.Gk, 75.30.Gw, 75.50.Gg

* e-mail: gkim@phy.sejong.ac.kr 
Consider a ferromagnetic film of thickness $z_{t}$ and its plane is perpendicular to the easy axis determined by the magnetocrystalline anisotropy energy depending on the crystal symmetry. Since the magnetic anisotropy energy has a time reversal symmetry in the absence of an external magnetic field, $\mathbf{M}$ has at least two easy directions. By applying a magnetic field $\mathbf{H}$ in a direction between perpendicular and opposite to the initial easy axis along which the system is magnetized, the symmetry of two equivalent orientations of $\mathbf{M}$ is broken. According to the classical theory of nucleation, [1] the switching of the magnetization occurs for a magnetic field greater than the critical field $H_{c}$ at which the spins of a saturated ferromagnet cease to be aligned. Besides the magnetic anisotropy energy $E_{a}$, the exchange energy $E_{e x}$ and the magnetic dipole-dipole interation $E_{d d}$ determine the switching process of the system. In this paper we simplify the system with small $E_{d d}$ compared with $E_{a}$ and $E_{e x}$. [2.33 At finite temperature, even for the field less than $H_{c}$, there is a nucleation due to thermal fluctuation whose rate is proportional to $\exp \left(-U / k_{B} T\right)$ where $U$ is an energy barrier related to the magnetic anisotropy energy, the external magnetic field and the exchange energy. At a temperature low enough to neglect the thermal activation process, the magnetic nucleation which is independent of temperature, may occur due to quantum tunneling. For the dynamical situation, it is important to include the effect of the environment on the quantum tunneling rate caused by phonons, [4] nuclear spins, [5] and Stoner excitations and eddy currents in metallic magnets. [6] However, many studies have shown that while these coupling might be crucial in macroscopic quantum coherence, they are not strong enough to make the quantum tunneling unobservable.

The question of the quantum nucleation were studied by Privorotskii [] who suggested the WKB exponent based on the dimensional analysis. Later, Chudnovsky and Gunther [2] proposed the quantum nucleation in a ferromagnetic thin film at zero temperature by using the instanton technique. In their work it was considered the system with the external magnetic field applied in the opposite direction to the initial easy axis, and presented the rate of quantum nucleation which allows experimental observation with a sufficient probability. In this paper we shall extend their considerations to the system with the magneic field applied 
in a range of angles $\pi / 2<\theta_{H} \leq \pi$ where $\theta_{H}$ is the angle between the initial easy axis ( $z$-axis) and the external magnetic field, which, in its method and spirit, is the generalization of Ref. [8] to problems with a non-uniform rotation of $\mathbf{M}$.

Using the parameter defined to be $\epsilon=1-H / H_{c}$, at zero temperature the nucleation exponent $B_{Q} \propto \epsilon^{3 / 4}$ in a wide range of angles, not $\epsilon^{1 / 2}$ which was obtained by Chudnovsky and Gunther for $\theta_{H}=\pi$. [2] The crossover temperature $T_{c}$ for quantum nucleation to thermal activation has a weak dependence on $\epsilon, T_{c} \propto \epsilon^{1 / 4}$ in a wide range of angle [8], compared with $\epsilon^{1 / 2}$ at $\theta_{H}=\pi$. [2] Also, it is found that the direction of $\mathbf{H}$ which favors the quantum nucleation lies in the range of angle $\pi / 2<\theta_{H}<\pi$, not $\pi$. In this paper we shall calculate instantons in the range of angles $\pi / 2+O(\sqrt{\epsilon})<\theta_{H}<\pi-O(\sqrt{\epsilon})$ at $T=0$ based on the imaginary time path integral method and their contribution to the quantum nucleation exponent $B_{Q}$, and discuss the $\theta_{H}$ dependence of $B_{Q}$ and the crossover temperature $T_{c}$ from quantum nucleation to thermally assisted process.

Our considerations begin by writing the transition amplitude in the imaginary time $\tau=i t$ from $-\tau_{I} / 2$ to $\tau_{I} / 2$ based on the spin coherent state path integral formalism

$$
\left\langle\hat{z}\left|\exp \left(-\tau_{I} H / \hbar\right)\right| \hat{z}\right\rangle=\int D[\mathbf{M}(\mathbf{r}, \tau)] \exp \left(-S_{E} / \hbar\right)
$$

where $\hat{z}$ is taken to be an initial easy direction of the magnetization, and $S_{E}$ is the Euclidean action which includes the Euclidean Lagrangian density $L_{E}$ as

$$
S_{E}=\int d \tau \int d^{3} \mathbf{r} L_{E}
$$

Since the magnitude of the total magnetization $\mathbf{M}$ is frozen up at sufficiently low temperature, the direction of the total magnetization becomes the only dynamical variable which we are interested in. In this situation the measure of the path integral $D[\mathbf{M}(\mathbf{r}, \tau)]$ in Eq. (西) is replaced by $D \Omega$ as

$$
D \Omega=\lim _{\epsilon \rightarrow 0} \prod_{k=1}^{N}\left(\frac{2 J+1}{4 \pi}\right) \sin \theta_{k} d \theta_{k} d \phi_{k}
$$


where $\epsilon=\max \left(\tau_{k+1}-\tau_{k}\right)$ and $J=M_{0} / \hbar \gamma$. Here $\gamma=g \mu_{B} / \hbar$, with $g$ being the g-factor, $\mu_{B}$ the Bohr magneton, $M_{0}$ the magnitude of the magnetization. Using the standard instanton method, the rate of quantum nucleation has the relation

$$
\Gamma_{Q} \propto \exp \left(-S_{E} / \hbar\right)
$$

and its Euclidean action for this problem is given by

$$
S_{E}=\int\left\{i \frac{M_{0}}{\gamma}[1-\cos \theta(\mathbf{r}, \tau)] \frac{d \phi(\mathbf{r}, \tau)}{d \tau}+E[\mathbf{M}(\mathbf{r}, \tau)]\right\} d \tau d^{3} \mathbf{r}
$$

The first term in Eq. (5) is the topological Wess-Zumino term [9], and the second term is the energy density which is composed of the magnetic anisotropy energy, the exchange energy and the energy given by an external magnetic field $\mathbf{H}$. So, the energy taken into account can be written as

$$
E[\mathbf{M}(\mathbf{r}, \tau)]=E_{a}+E_{e x}-\mathbf{M} \cdot \mathbf{H}
$$

We notice that from $\delta S_{E}=0$ the action (5) produces the classical equation of motion 10 whose solution is called instanton, [11] and describes the $1 \oplus 1$ dimensional dynamics in the Hamiltonian formulation, which consists of the canonical coordinates $\phi$ and $p_{\phi}=1-\cos \theta$. 12,13

In this paper we study the biaxial symmetry whose magnetic anisotropy energy is given by

$$
E_{a}=K_{1}\left(\alpha_{1}^{2}+\alpha_{2}^{2}\right)+K_{2} \alpha_{2}^{2}
$$

where $K_{1}$ and $K_{2}$ are the parallel and transverse anisotropy constants, and $\alpha_{1}, \alpha_{2}$, and $\alpha_{3}$ the directional cosines. The exchange interaction is expressed as [1]

$$
E_{e x}=\frac{1}{2} C\left[\left(\nabla \alpha_{1}\right)^{2}+\left(\nabla \alpha_{2}\right)^{2}+\left(\nabla \alpha_{3}\right)^{2}\right]
$$

where $C$ is an exchange constant. Applying the magnetic field in the $x z$-plane [8 and expressing $\alpha_{1}, \alpha_{2}$ and $\alpha_{3}$ in terms of the fields $\theta(\mathbf{r}, \tau)$ and $\phi(\mathbf{r}, \tau)$ which are spherical coordinates of $\mathbf{M}$, the total energy (6) is given by 


$$
\begin{aligned}
E[\theta(\mathbf{r}, \tau), \phi(\mathbf{r}, \tau)]= & K_{1} \sin ^{2} \theta+K_{2} \sin ^{2} \phi \sin ^{2} \theta+\frac{1}{2} C\left[(\nabla \theta)^{2}+(\nabla \phi)^{2} \sin ^{2} \theta\right] \\
& -H_{x} M_{0} \sin \theta \cos \phi-H_{z} M_{0} \cos \theta+E_{0}
\end{aligned}
$$

where $E_{0}$ is introduced to make $E(\theta, \phi)$ zero at the initial orientation. As will be seen later, the effective mass of the magnetic particle is inversely proportional to a linear combination of $K_{2}$ and $H_{x}$ while there is no exact analog of the kinetic energy in the action (5). For this reason we need either the transverse anisotropy constant $K_{2}$ or the magnetic field $H_{x}$ transverse to the initial easy axis for quantum nucleation. Introducing the dimensionless constants,

$$
\bar{K}_{2}=K_{2} / 2 K_{1}, \quad \bar{C}=C / 4 K_{1}, \quad \bar{H}_{x}=H_{x} / H_{0}, \quad \bar{H}_{z}=H_{z} / H_{0}
$$

where $H_{0}=2 K_{1} / M_{0}$, we obtain the total energy (9) written as

$$
\begin{aligned}
\bar{E}[\theta(\mathbf{r}, \tau), \phi(\mathbf{r}, \tau)]= & \frac{1}{2} \sin ^{2} \theta+\bar{K}_{2} \sin ^{2} \phi \sin ^{2} \theta+\bar{C}\left[(\nabla \theta)^{2}+(\nabla \phi)^{2} \sin ^{2} \theta\right] \\
& -\bar{H}_{x} \sin \theta \cos \phi-\bar{H}_{z} \cos \theta+\bar{E}_{0}
\end{aligned}
$$

where $\bar{E}(\theta, \phi)=E(\theta, \phi) / 2 K_{1}$. The plane given by $\phi=0$ is the easy plane, on which $\bar{E}(\theta, \phi)$ is given by

$$
\bar{E}(\theta, 0)=\frac{1}{2} \sin ^{2} \theta-\bar{H} \cos \left(\theta-\theta_{H}\right)+\bar{E}_{0}
$$

where we used $\bar{H}_{x}=\bar{H} \sin \theta_{H}$ and $\bar{H}_{z}=\bar{H} \cos \theta_{H}$ since $\mathbf{H}$ lies in $x z$-plane. Let us define $\theta_{0}$ to be the angle of metastable state generated by the anisotropy energy and the external magnetic field, and $\theta_{c}$ the angle at which the barrier vanishes by the applied critical magnetic field $H_{c}$. Then, $\theta_{0}$ is determined by $[d \bar{E}(\theta, 0) / d \theta]_{\theta=\theta_{0}}=0$, and $\theta_{c}$ and $\bar{H}_{c}$ by $[d \bar{E}(\theta, 0) / d \theta]_{\theta=\theta_{c}, \bar{H}=\bar{H}_{c}}=0$ and $\left[d^{2} \bar{E}(\theta, 0) / d \theta^{2}\right]_{\theta=\theta_{c}, \bar{H}=\bar{H}_{c}}=0$. After a little calculations, the dimensionless critical field $\bar{H}_{c}$ and the critical angle $\theta_{c}$ are expressed as [13,8]

$$
\begin{aligned}
\bar{H}_{c} & =\left(\sin ^{2 / 3} \theta_{H}+\left|\cos \theta_{H}\right|^{2 / 3}\right)^{-3 / 2}, \\
\theta_{c} & =\frac{1}{2} \arcsin \left[\frac{2\left|\cot \theta_{H}\right|^{1 / 3}}{1+\left|\cot \theta_{H}\right|^{2 / 3}}\right] .
\end{aligned}
$$


For, e.g., $\theta_{H}=3 \pi / 4$ and $\pi$, we have $\theta_{c}=\pi / 4$ and 0 , respectively. Simple analysis for Eq. (12) shows that, since the height and the width of the barrier are proportional to the power of the parameter $\epsilon$, the value of $\epsilon$ should be small in order to have a large tunneling rate. The small value of $\epsilon$ can be achieved by tuning $H$ to $H_{c}$. From now on we will consider the value of $\epsilon$ to be small, that is to say, $\epsilon \ll 1$. From the equations which $\theta_{0}$ and $\theta_{c}$ satisfy, we obtain the equation for $\eta\left(=\theta_{c}-\theta_{0}\right)$ as [13]

$$
\sin \left(2 \theta_{c}\right)\left(\epsilon-\frac{3}{2} \eta^{2}\right)-\eta \cos \left(2 \theta_{c}\right)\left(2 \epsilon-\eta^{2}\right)=0
$$

Simple calculations show that $\eta$ is of the order of $\sqrt{\epsilon}$. Thus the order of magnitude of the second term in Eq. (15) is smaller than that of the first term by $\sqrt{\epsilon}$ and the value of $\eta$ is determined by the first term which leads to $\eta \simeq \sqrt{2 \epsilon / 3}$. However, when $\theta_{H}$ is very close to $\pi / 2$ or $\pi, \sin \left(2 \theta_{c}\right)$ becomes almost zero, as shown in Fig. [1, and the first term is much smaller than the second term in Eq. (15). Therefore the value of $\eta$ is obtained from the second term when $\theta_{H} \simeq \pi / 2$ or $\pi$, which leads to $\eta \simeq \sqrt{2 \epsilon}$ for $\theta_{H} \simeq \pi / 2$ and $\eta \simeq 0$ for $\theta_{H} \simeq \pi$ from the detailed analysis of the equations which $\theta_{0}$ and $\theta_{c}$ satisfy. Since the first term in (15) is dominant in the range of value $\theta_{c}$ which satisfies $\tan \left(2 \theta_{c}\right)>O(\sqrt{\epsilon}), \eta \simeq \sqrt{2 \epsilon / 3}$ is valid for $\pi / 2+O(\sqrt{\epsilon})<\theta_{H}<\pi-O(\sqrt{\epsilon})$ by usnig Eq. (14). As is shown in Fig. 2, this is checked by performing the numerical calculation for the equations which $\theta_{0}$ and $\theta_{c}$ satisfy. From Eq. (11) we obtain an approximate form of $\bar{E}(\theta, \phi)$ as

$$
\begin{aligned}
\bar{E}(\delta, \phi)= & \bar{K}_{2} \sin ^{2} \phi \sin ^{2}\left(\theta_{0}+\delta\right)+\bar{H}_{x} \sin \left(\theta_{0}+\delta\right)(1-\cos \phi) \\
& +\bar{C}\left[(\nabla \delta)^{2}+(\nabla \phi)^{2} \sin ^{2}\left(\theta_{0}+\delta\right)\right]+\bar{E}_{1}(\delta)
\end{aligned}
$$

where $\bar{E}(\theta, \phi)$ is written as $\bar{E}(\delta, \phi)$ by introducing a small variable $\delta=\theta-\theta_{0}$, and $\bar{E}_{1}(\delta)$ is a function of only $\delta$ given by

$$
\bar{E}_{1}(\delta)=\frac{1}{4} \sin \left(2 \theta_{c}\right)\left(3 \delta^{2} \eta-\delta^{3}\right)+\frac{1}{2} \cos \left(2 \theta_{c}\right)\left[\delta^{2}\left(\epsilon-\frac{3}{2} \eta^{2}\right)+\delta^{3} \eta-\frac{\delta^{4}}{4}\right] .
$$

As previously considered for $\eta$ in Eq. (15), even though the $\cos \left(2 \theta_{c}\right)$-term in Eq. (17) looks smaller by a factor of $\eta$ which is of the order of $\sqrt{\epsilon}$, the second term can not be neglected 
near $\theta_{H}=\pi / 2$ and $\pi$ because $\sin \left(2 \theta_{c}\right)$ is almost zero for these regions of $\theta_{H}$. In order to evaluate the order of magnitude of each term in the action (5) and simplify the calculations for $\epsilon \ll 1$, it is convenient to use new scaled variables

$$
\tilde{\tau}=\epsilon^{\frac{\alpha}{2}} \omega_{0} \tau, \quad \tilde{\mathbf{r}}=\epsilon^{\frac{\beta}{2}} \mathbf{r} / r_{0}, \quad \bar{\delta}=\delta / \sqrt{\epsilon}, \quad r_{0}=\sqrt{2 \bar{C}}, \quad \omega_{0}=2 \gamma K_{1} / M_{0}
$$

where it is noted that $r_{0}$ is the thickness of the domain wall in a bulk ferromagnet. Then, the Euclidean action (5) becomes

$$
\begin{aligned}
S_{E}[\delta(\tilde{\mathbf{r}}, \tilde{\tau}), \phi(\tilde{\mathbf{r}}, \tilde{\tau})]= & \hbar J \epsilon^{-\frac{\alpha+3 \beta}{2}} r_{0}^{3} \int d \tilde{\tau} d^{3} \tilde{\mathbf{r}}\left\{i \epsilon^{\frac{\alpha}{2}}\left[1-\cos \left(\theta_{0}+\sqrt{\epsilon} \bar{\delta}\right)\right] \frac{\partial \phi}{\partial \tilde{\tau}}\right. \\
& +\bar{K}_{2} \sin ^{2} \phi \sin ^{2}\left(\theta_{0}+\sqrt{\epsilon} \bar{\delta}\right)+\bar{H}_{x} \sin \left(\theta_{0}+\sqrt{\epsilon} \bar{\delta}\right)(1-\cos \phi) \\
& +\frac{1}{2} \epsilon^{1+\beta}(\tilde{\nabla} \bar{\delta})^{2}+\frac{1}{2} \epsilon^{\beta} \sin ^{2}\left(\theta_{0}+\sqrt{\epsilon} \bar{\delta}\right)(\tilde{\nabla} \phi)^{2} \\
& \left.+\frac{1}{4} \sin \left(2 \theta_{c}\right) \epsilon^{\frac{3}{2}}\left(3 \bar{\delta}^{2} \frac{\eta}{\sqrt{\epsilon}}-\bar{\delta}^{3}\right)+\frac{1}{2} \cos \left(2 \theta_{c}\right) \epsilon^{2}\left[\bar{\delta}^{2}\left(1-\frac{3 \eta^{2}}{2 \epsilon}\right)+\bar{\delta}^{3} \frac{\eta}{\sqrt{\epsilon}}-\frac{\bar{\delta}^{4}}{4}\right]\right\} .
\end{aligned}
$$

where the parameters $\alpha$ and $\beta$ are fixed by the analysis of the order of magnitude of each term in the action (19) subject to the situation considered.

For $\pi / 2+O(\sqrt{\epsilon})<\theta_{H}<\pi-O(\sqrt{\epsilon})$, the critical angle $\theta_{c}$ and the angle of metastable state $\theta_{0}$ are in the range of angle from 0 to $\pi / 2$, and $\eta \simeq \sqrt{2 \epsilon / 3}$. In this situation, the action (19) becomes

$$
\begin{aligned}
S_{E}[\delta(\tilde{\mathbf{r}}, \tilde{\tau}), \phi(\tilde{\mathbf{r}}, \tilde{\tau})]= & \hbar J \epsilon^{-\frac{\alpha+3 \beta}{2}} r_{0}^{3} \int d \tilde{\tau} d^{3} \tilde{\mathbf{r}}\left\{-i \epsilon^{\frac{\alpha+1}{2}} \sin \left(\theta_{0}+\sqrt{\epsilon} \bar{\delta}\right) \phi\left(\frac{\partial \bar{\delta}}{\partial \tilde{\tau}}\right)\right. \\
& +\bar{K}_{2} \sin ^{2} \phi \sin ^{2}\left(\theta_{0}+\sqrt{\epsilon} \bar{\delta}\right)+2 \bar{H}_{x} \sin \left(\theta_{0}+\sqrt{\epsilon} \bar{\delta}\right) \sin ^{2}\left(\frac{\phi}{2}\right) \\
& +\frac{1}{2} \epsilon^{1+\beta}(\tilde{\nabla} \bar{\delta})^{2}+\frac{1}{2} \epsilon^{\beta} \sin ^{2}\left(\theta_{0}+\sqrt{\epsilon} \bar{\delta}\right)(\tilde{\nabla} \phi)^{2} \\
& \left.+\frac{1}{4} \sin \left(2 \theta_{c}\right) \epsilon^{\frac{3}{2}}\left(3 \bar{\delta}^{2} \frac{\eta}{\sqrt{\epsilon}}-\bar{\delta}^{3}\right)\right\}
\end{aligned}
$$

where we performed the integration by part for the first term and neglected the total time derivative. Since the last term in the action (20) makes the potential barrier in our problem, the order of magnitude of each term should be the same order as that of the last term. Since the order of magnitude of each term is $O\left(\epsilon^{(\alpha+1) / 2} \phi\right), O\left(\sin ^{2} \phi\right), O\left(\sin ^{2}(\phi / 2)\right), \epsilon^{1+\beta}, O\left(\epsilon^{\beta} \phi^{2}\right)$ 
and $\epsilon^{3 / 2}$, respectively, the small values of $\phi$ contribute to the path integral for $\epsilon \ll 1$, and its order of magnitude is expected to be $\epsilon^{3 / 4}$. Choosing $\alpha=\beta=1 / 2$, the order of magnitude of the term which includes $(\tilde{\nabla} \phi)^{2}$ is $\epsilon^{2}$ while those of the other terms are $\epsilon^{3 / 2}$. Thus, the fifth term in Eq. (20) is higher and neglected.

Performing the Gaussian integration over $\phi$ in the transition amplitude (11) and the measure (3), the remaining integral is of the form

$$
\int D[\delta(\tilde{\mathbf{r}}, \tilde{\tau})] \exp \left(-S_{E}^{e f f} / \hbar\right)
$$

where from Eq. (20) the effective action is given by

$$
S_{E}^{e f f}[\delta(\tilde{\mathbf{r}}, \tilde{\tau})]=\hbar J r_{0}^{3} \epsilon^{\frac{1}{2}} \int d \tilde{\tau} d^{3} \tilde{\mathbf{r}}\left[\frac{1}{2} M\left(\frac{\partial \bar{\delta}}{\partial \tilde{\tau}}\right)^{2}+\frac{1}{2}(\tilde{\nabla} \bar{\delta})^{2}+\frac{1}{4} \sin \left(2 \theta_{c}\right)\left(\sqrt{6} \bar{\delta}^{2}-\bar{\delta}^{3}\right)\right]
$$

Here the effective mass in the reduced dimension is given by

$$
M=\frac{\sin \theta_{c}}{\bar{H}_{x}+2 \bar{K}_{2} \sin \theta_{c}} .
$$

Introducing the variables $\bar{\tau}=\tilde{\tau} \sqrt{\sin \left(2 \theta_{c}\right) / M}$ and $\overline{\mathbf{r}}=\tilde{\mathbf{r}} \sqrt{\sin \left(2 \theta_{c}\right)}$, we simplify the effective action (22) to be

$$
S_{E}^{e f f}[\delta(\overline{\mathbf{r}}, \bar{\tau})]=\hbar J r_{0}^{3} \epsilon^{\frac{1}{2}} \frac{\sqrt{M}}{\sin \left(2 \theta_{c}\right)} \int d \bar{\tau} d^{3} \overline{\mathbf{r}}\left[\frac{1}{2}\left(\frac{\partial \bar{\delta}}{\partial \bar{\tau}}\right)^{2}+\frac{1}{2}(\bar{\nabla} \bar{\delta})^{2}+\frac{1}{4}\left(\sqrt{6} \bar{\delta}^{2}-\bar{\delta}^{3}\right)\right]
$$

In case of a small magnetic particle of volume $V \ll r_{0}^{3}, \bar{\delta}$ does not depend on the space $\overline{\mathbf{r}}$, which leads to the action

$$
S_{E}^{e f f}[\delta(\bar{\tau})]=\hbar J \epsilon^{\frac{5}{4}} V \sqrt{M \sin \left(2 \theta_{c}\right)} \int d \bar{\tau}\left[\frac{1}{2}\left(\frac{d \bar{\delta}}{d \bar{\tau}}\right)^{2}+\frac{1}{4}\left(\sqrt{6} \bar{\delta}^{2}-\bar{\delta}^{3}\right)\right]
$$

The corresponding classical trajectory is given by

$$
\bar{\delta}_{c l}(\bar{\tau})=\frac{\sqrt{6}}{\cosh ^{2}\left(\left(\frac{3}{32}\right)^{1 / 4} \bar{\tau}\right)}
$$

and its classical action is found to be 13

$$
S_{c l}=(\hbar J) \frac{16 \times 6^{1 / 4}}{5} \epsilon^{5 / 4} \frac{\left|\cot \theta_{H}\right|^{1 / 6}}{\sqrt{1+\frac{K_{2}}{K_{1}}\left(1+\left|\cot \theta_{H}\right|^{2 / 3}\right)}} .
$$


The geometry of the problem in this paper is the thin film of thickness $z_{t}$ less than the size $r_{0} / \epsilon^{1 / 4}$ of the critical nucleus and its plane is perpendicular to the initial easy axis. After performing the integration over $\bar{z}$-variable, the action (24) becomes $S_{E}^{e f f}[\delta(\overline{\mathbf{r}}, \bar{\tau})]=\hbar J r_{0}^{2} z_{t} \epsilon^{\frac{3}{4}} \sqrt{\frac{M}{\sin \left(2 \theta_{c}\right)}} \int d \bar{\tau} d^{2} \overline{\mathbf{r}}\left[\frac{1}{2}\left(\partial_{\bar{\tau}} \bar{\delta}\right)^{2}+\frac{1}{2}\left(\partial_{\bar{x}} \bar{\delta}\right)^{2}+\frac{1}{2}\left(\partial_{\bar{y}} \bar{\delta}\right)^{2}+\frac{1}{4}\left(\sqrt{6} \bar{\delta}^{2}-\bar{\delta}^{3}\right)\right]$,

At zero temperature the classical solution of the action (28) has $O(3)$ symmetry in $\bar{\tau}$ and $\overline{\mathbf{r}}$. Therefore, $\bar{\delta}$ is a function of $q$ where $q=\left(\bar{x}^{2}+\bar{y}^{2}+\bar{\tau}^{2}\right)^{1 / 2}$, in which the effective action (28) becomes

$$
S_{E}^{e f f}[\delta(\overline{\mathbf{r}}, \bar{\tau})]=4 \pi \hbar J r_{0}^{2} z_{t} \epsilon^{\frac{3}{4}} f\left(\theta_{H}\right) \int_{0}^{\infty} d q q^{2}\left[\frac{1}{2}\left(\frac{d \bar{\delta}}{d q}\right)^{2}+\frac{1}{4}\left(\sqrt{6} \bar{\delta}^{2}-\bar{\delta}^{3}\right)\right]
$$

with the angular dependence

$$
f\left(\theta_{H}\right)=\frac{1+\left|\cot \theta_{H}\right|^{2 / 3}}{\sqrt{2}\left|\cot \theta_{H}\right|^{1 / 6} \sqrt{1-\epsilon+\frac{K_{2}}{K_{1}}\left(1+\left|\cot \theta_{H}\right|^{2 / 3}\right)}} .
$$

The corresponding classical equation of motion satisfies

$$
\frac{d^{2} \bar{\delta}}{d q^{2}}+\frac{2}{q} \frac{d \bar{\delta}}{d q}=\frac{\sqrt{6}}{2} \bar{\delta}-\frac{3}{4} \bar{\delta}^{2}
$$

This equation can be solved by the numerical approach, whose results are similar to the ones in Ref. [2]. The maximal value of $\bar{\delta}$ is 6.85 at $\bar{\tau}=0$ and $\bar{r}=0$. Using the results, we obtain the rate of quantum nucleation given by

$$
\Gamma_{Q} \propto \exp \left(-S_{E} / \hbar\right)=\exp \left[-105.2 J r_{0}^{2} f\left(\theta_{H}\right) z_{t} \epsilon^{3 / 4}\right]
$$

Since the period $\tau_{I}$ of the instanton becomes zero for the temperature much larger than $T_{c}$, the instanton solution becomes independent of $\bar{\tau}$. In this case we have the rate of thermal nucleation

$$
\Gamma_{T} \propto \exp \left(-\frac{U^{e f f}}{k_{B} T}\right)
$$

where the effective height of the barrier 


$$
U^{e f f}=4 \pi K_{1} r_{0}^{2} z_{t} \epsilon \int_{0}^{\infty} d q q\left[\frac{1}{2}\left(\frac{d \bar{\delta}}{d q}\right)^{2}+\frac{\sqrt{6}}{4} \bar{\delta}^{2}-\frac{1}{4} \bar{\delta}^{3}\right]
$$

From the saddle point of the functional the shape of thermal nucleus is determined by

$$
\frac{d^{2} \bar{\delta}}{d q^{2}}+\frac{1}{q} \frac{d \bar{\delta}}{d q}-\frac{\sqrt{6}}{2} \bar{\delta}+\frac{3}{4} \bar{\delta}^{2}=0
$$

The solution is found by the numerical method whose shape is similar to the one in Ref. [2]. The maximal size of the thermal nucleus is 3.9 at $\overline{\mathbf{r}}=0$. Using this result, the effective height of the barrier becomes

$$
U^{e f f}=41.3 K_{1} r_{0}^{2} z_{t} \epsilon
$$

Comparing this with Eq. (32), we obtain the approximate form of the temperature of the crossover from thermal to quantum regime given by

$$
T_{c} \approx 0.22 \frac{K_{1} \epsilon^{1 / 4}}{k_{B} J f\left(\theta_{H}\right)} .
$$

Here we notice that the angular and the field dependence of $T_{c}$ coincides with the one of Ref. [13] and with that of Ref. [8] in the limit of $\bar{K}_{2}=0$. It is understood from the fact that $T_{c} \sim \hbar \omega_{b} / k_{B}$, where the frequency $\omega_{b}$ of small oscillations around the minimum of the inverted potential is proportional to $\omega_{0} \epsilon^{1 / 4} / f\left(\theta_{H}\right)$ from Eq. (18) and the scaling relation between $\bar{\tau}$ and $\tilde{\tau}$.

Let us now consider the situation with the magentic field opposite to the initial easy axis. For $\theta_{H}=\pi$, we obtain $\theta_{c}=0$ and $\eta=0$ by using Eqs. (14) and (15). In this case, noting that $\theta_{0}=0, \bar{H}_{x}=0$ and $\epsilon \ll 1$, the Euclidean action (19) can be simplified to be

$$
\begin{aligned}
S_{E}[\delta(\tilde{\mathbf{r}}, \tilde{\tau}), \phi(\tilde{\mathbf{r}}, \tilde{\tau})]= & \hbar J \epsilon^{-\frac{\alpha+3 \beta}{2}} r_{0}^{3} \int d \tilde{\tau} d^{3} \tilde{\mathbf{r}}\left[\frac{i}{2} \epsilon^{1+\frac{\alpha}{2}} \bar{\delta}^{2} \frac{\partial \phi}{\partial \tilde{\tau}}+\bar{K}_{2} \epsilon \bar{\delta}^{2} \sin ^{2} \phi\right. \\
& \left.+\frac{1}{2} \epsilon^{1+\beta}(\tilde{\nabla} \bar{\delta})^{2}+\frac{1}{2} \epsilon^{1+\beta} \bar{\delta}^{2}(\tilde{\nabla} \phi)^{2}+\frac{1}{2} \epsilon^{2}\left(\bar{\delta}^{2}-\frac{\bar{\delta}^{4}}{4}\right)\right] .
\end{aligned}
$$

In order to obtain the quantum tunneling under the barrier, we need the second term which includes the transverse component of the anisotropy energy $E_{a}$. Without $K_{2}$ in $E_{a}$, $M_{z}$, as a quantum operator, commute with $E_{a}$ and conserves. Thus, $K_{2}$ is responsible for 
quantum transition at $\theta_{H}=\pi$. Comparing the second term with the last term which makes the potential barrier, only small values of $\phi$ contribute to the path integral because $\epsilon \ll 1$. Thus we expect the order of magnitude of $\phi$ to be $\epsilon^{1 / 2}$. Choosing $\alpha=\beta=1$, the order of magnitude of each term except the fourth term in Eq. (38) is $\epsilon^{2}$ while the fourth one is $\epsilon^{3}$ which is higher and neglected. Following the same procedure as we have done previously, we obtained the WKB exponent $B_{Q}\left(T=0, \theta_{H}=\pi\right)$, the effective height of the barrier $U^{e f f}\left(\theta_{H}=\pi\right)$ for $T>T_{c}$ and the approximate form of the crossover temperature $T_{c}$, which were presented by Chudnovsky and Gunther [2]. Recently, Ferrera and Chudnovsky discussed the thermally assisted quantum nucleation at $\theta_{H}=\pi$ and obtained the temperature dependence of the WKB exponent $B\left(T, \theta_{H}=\pi\right)$ in the range of temperature $0<T<2 T_{c}$ by numerically solving the partial differential equation with period $\hbar \omega \epsilon^{1 / 2} / T$ where $\omega=\left(2 \gamma / M_{0}\right) \sqrt{K_{1} K_{2}}$.

As summarized in Table $\mathbb{E}, B_{Q}$ is proportional to $\epsilon^{3 / 4}$ for a range of angles $\pi / 2+O(\sqrt{\epsilon})<$ $\theta_{H}<\pi-O(\sqrt{\epsilon})$, while $B_{Q} \propto \epsilon^{1 / 2}$ at $\theta_{H}=\pi$. By using the interpolation method, we obtain $B_{Q}$ in the range of angle close to $\pi$, and present the results in Fig. 3 for $\epsilon=0.01$. In case of volume $V \ll r_{0}^{3}$ [8, [13], $B_{Q}$ can be minimum at $\theta_{H}=\pi$ depending on the ratio $K_{2} / K_{1}$ of the anisotropy constants. $B_{Q}\left(\theta_{H}=\pi\right)$ is minimum for $K_{2}=K_{1}$ while it is maximum in the limit of $K_{2} \ll K_{1}$ which is uniaxial symmetry [8]. In the ferromagetic film which we have considered with small $\epsilon, B_{Q}$ has a minimum in the range of angle $\pi / 2+O(\sqrt{\epsilon})<\theta_{H}<\pi-O(\sqrt{\epsilon})$ for both $K_{2}=K_{1}$ and $K_{2} \ll K_{1}$, as is shown in Table घ. Also, in the limit of $K_{2} \gg K_{1}$ the dependence of $f\left(\theta_{H}\right)$ on the ratio $K_{2} / K_{1}$ is the same as that of $B_{Q}$ at $\theta_{H}=\pi$. Thus, in the small $\epsilon$ limit, the ratio does not make an effect on the shape of $B_{Q}$, which is different from the situation for $V \ll r_{0}^{3}$. [13 In other words, since $B_{Q}$ has a shape similar to Fig. 3, it always has a minimum in the range of angle $\pi / 2+O(\sqrt{\epsilon})<\theta_{H}<\pi-O(\sqrt{\epsilon})$. The minimal value of $B_{Q}$ or equivalently the maximal value of $\Gamma_{Q}$ at $T=0$ can be obtained by the analysis of $f\left(\theta_{H}\right)$ and its $\theta_{H}$ is given by

$$
\theta_{H}=\pi-\arctan \left[\frac{2 \bar{K}_{2}}{\sqrt{\left(2 \bar{K}_{2}+\frac{1}{2}\right)^{2}+2}-\frac{3}{2}}\right]^{3 / 2}
$$


where we notice that $\theta_{H} \approx 1.97\left(\simeq 112.9^{\circ}\right)$ for, e.g., $K_{1}=K_{2}$, as is shown in Fig. 3. Simple analysis shows that the angle which gives the minimal valus of $B_{Q}$, Eq. (39), decreases as $\bar{K}_{2}$ decreases, and converges to $\pi-\arctan (3 \sqrt{3})\left(\approx 1.76 \simeq 100.9^{\circ}\right)$ in the limit of $\bar{K}_{2}=0$. [8] Since the effective height of barrier $U^{e f f}$ is proportional to $\epsilon$ for both $\pi / 2+O(\sqrt{\epsilon})<$ $\theta_{H}<\pi-O(\sqrt{\epsilon})$ and $\theta_{H}=\pi$, we obtain $T_{c} \propto \epsilon^{1 / 4}$ for $\pi / 2+O(\sqrt{\epsilon})<\theta_{H}<\pi-O(\sqrt{\epsilon})$ and $T_{c} \propto \epsilon^{1 / 2}$ for $\theta_{H}=\pi$. Since $T_{c}$ is inversely proportional to $f\left(\theta_{H}\right)$ for a wide range of angles, $T_{c}$ becomes maximum at the angle (39). For $K_{1} \simeq K_{2} \simeq 10^{7} \mathrm{erg} / \mathrm{cm}^{3}, M_{0} \simeq 500 \mathrm{emu} / \mathrm{cm}^{3}$ and $\epsilon \sim 10^{-2}-10^{-3}$, the maximal value of $T_{c}$ is approximately $235-132 m K$ while $T_{c} \approx$ $167-53 m K$ for $\theta_{H}=\pi$.

In conclusion, we have studied the quantum nucleation of a thin ferromagnetic film placed at some angle with the magnetic field and presented the quantum nucleation exponent and the crossover temperature based on the spin coherent path integral formalism. If the experiment is to be performed, there are three control parameters for comparison with theory: the angle of the magnetic field $\theta_{H}$, the magnitude of the field $\mathbf{H}$ in terms of $\epsilon$, and the temperature $T$. The dependence of $B_{Q}$ and $T_{c}$ on the power of $\epsilon$ seems to be observed by changing $\theta_{H}$. Also, the angle (39) which gives the maximal value of $T_{c}$ as well as $\Gamma_{Q}$ is expected to be observed in experiment.

Discussions with D. S. Hwang are gratefully acknowledged. This work was supported in part by the Basic Science Research Institute Program, Ministry of Education, Project No. BSRI-96-2414, in part by the Ministry of Science and Technology of Korea through the HTSRA, and in part by Non-Directed-Research-Fund, Korea Research Foundation 1996. 


\section{REFERENCES}

[1] E. H. Frei, S. Shtrikman and D. Treves, Phys. Rev. 106, 446 (1957).

[2] E. M. Chudnovsky and L. Gunther, Phys. Rev. B 37 (1988).

[3] A. Ferrera and E. M. Chudnovsky, Phys. Rev. B 53, 354 (1996).

[4] Anupam Garg and Gwang-Hee Kim, Phys. Rev. Lett. 63, 2512 (1989); Phys. Rev. B 43, 712 (1991); H. Simanjuntak, J. Low. Temp. Phys. 90, 405 (1992).

[5] Anupam Garg, Phys. Rev. Lett. 70, 1541 (1993).

[6] G. Tatara and H. Fukuyama, Phys. Rev. Lett. 72 , 772 (1994).

[7] I. A. Privorotskii, Sov. Phys. Usp. 15, 555 (1973).

[8] M.-C. Miguel and E. M. Chudnovsky, Phys. Rev. B 54, 388 (1996).

[9] D. Loss, D. P. DiVincenzo, and G. Grinstein, Phys. Rev. Lett. 69, 3232 (1992); J. von Delft and C. L. Henley, Phys. Rev. Lett. 69, 3236 (1992); E. Fradkin and M. Stone, Phys. Rev. B 38, 7215 (1988).

[10] T. L. Gilbert, Phys. Rev. 100, 1243 (1955); W. F. Brown, Jr. Magnetostatic Principles in Ferromagnetism (North-Holland, Amsterdam, 1962); V. G. Bar'yakhter, M. V. Chetkin, B. A. Ivanov, and S. N. Gadetskii, Dynamics of Topological Magnetic Solitons, (Springer-Verlag, Berlin, 1994).

[11] J. S. Langer, Ann. Phys.(NY) 41, 108 (1967); S. Coleman, Aspects of Symmetry (Cambridge University Press, 1985), Chap. 7.

[12] K. Johnson, Ann. Phys. 192, 104 (1989); H. B. Nielsen and D. Rohrlich, Nucl. Phys. B 299, 471 (1988) ; J.-Q. Liang, H. J. W. Muller-Kirsten, J.-G. Zhou, F. Zimmerchied, and F.-C. Pu, hep-th-9612106.

[13] Gwang-Hee Kim and Dae Sung Hwang (to be published). 


\section{TABLES}

TABLE I. Summary of the results for the quantum nucleation in the range of angle $\pi / 2+O(\sqrt{\epsilon})<\theta_{H}<\pi-O(\sqrt{\epsilon})$ and $\theta_{H}=\pi$. Here $B_{Q}$ is the WKB exponent at $T=0$ and $f\left(\theta_{H}\right)$ given by Eq. (30).

\begin{tabular}{|c|c|c|}
\hline Field angle & $\pi / 2+O(\sqrt{\epsilon})<\theta_{H}<\pi-O(\sqrt{\epsilon})$ & $\theta_{H}=\pi$ [2] \\
\hline$\alpha=\beta$ & $1 / 2$ & 1 \\
$M$ & $\left(2 \bar{K}_{2}+\bar{H}_{x} / \sin \theta_{c}\right)^{-1}$ & $\left(4 \bar{K}_{2}\right)^{-1}$ \\
$B_{Q} / J r_{0}^{2} z_{t}$ & $105.2 f\left(\theta_{H}\right) \epsilon^{3 / 4}$ & $37.9 \sqrt{K_{1} / K_{2}} \epsilon^{1 / 2}$ \\
$U^{e f f} / K_{1} r_{0}^{2} z_{t}$ & $41.3 \epsilon$ & $23.4 \epsilon$ \\
$\left(k_{B} T_{c}\right) J / K_{1}$ & $0.22 \epsilon^{1 / 4} / f\left(\theta_{H}\right)$ & $0.62 \sqrt{K_{2} / K_{1}} \epsilon^{1 / 2}$ \\
\hline
\end{tabular}




\section{FIGURES}

FIG. 1. Comparison of (a) $\sin \left(2 \theta_{c}\right)$ with (b) $\cos \left(2 \theta_{c}\right)$ in Eqs. (15) and (17). Note that $\sin \left(2 \theta_{c}\right)=0,\left|\cos \left(2 \theta_{c}\right)\right|=1$ for both $\theta_{H}=\pi / 2$ and $\pi$.

FIG. 2. The shape of $\eta\left(=\theta_{c}-\theta_{0}\right)$ as a function of $\theta_{H}$ for (a) $\epsilon=0.01$ and (b) $\epsilon=0.001$. Note that $\eta \approx \sqrt{2 \epsilon / 3}$ is valid in a wide range of angles, i.e., $\pi / 2+O(\sqrt{\epsilon})<\theta_{H}<\pi-O(\sqrt{\epsilon})$.

FIG. 3. The $\theta_{H}$ dependence of the quantum nucleation exponent $\bar{B}_{Q}$ for $K_{1}=K_{2}$ and $\epsilon=0.01$, where $\bar{B}_{Q}=B_{Q} / J r_{0}^{2} z_{t}$. The minimal value of $\bar{B}_{Q}$ is approximately 2.65 at $\theta_{H} \approx 1.97$ while $\bar{B}_{Q} \approx 3.79$ at $\theta_{H}=\pi$. 


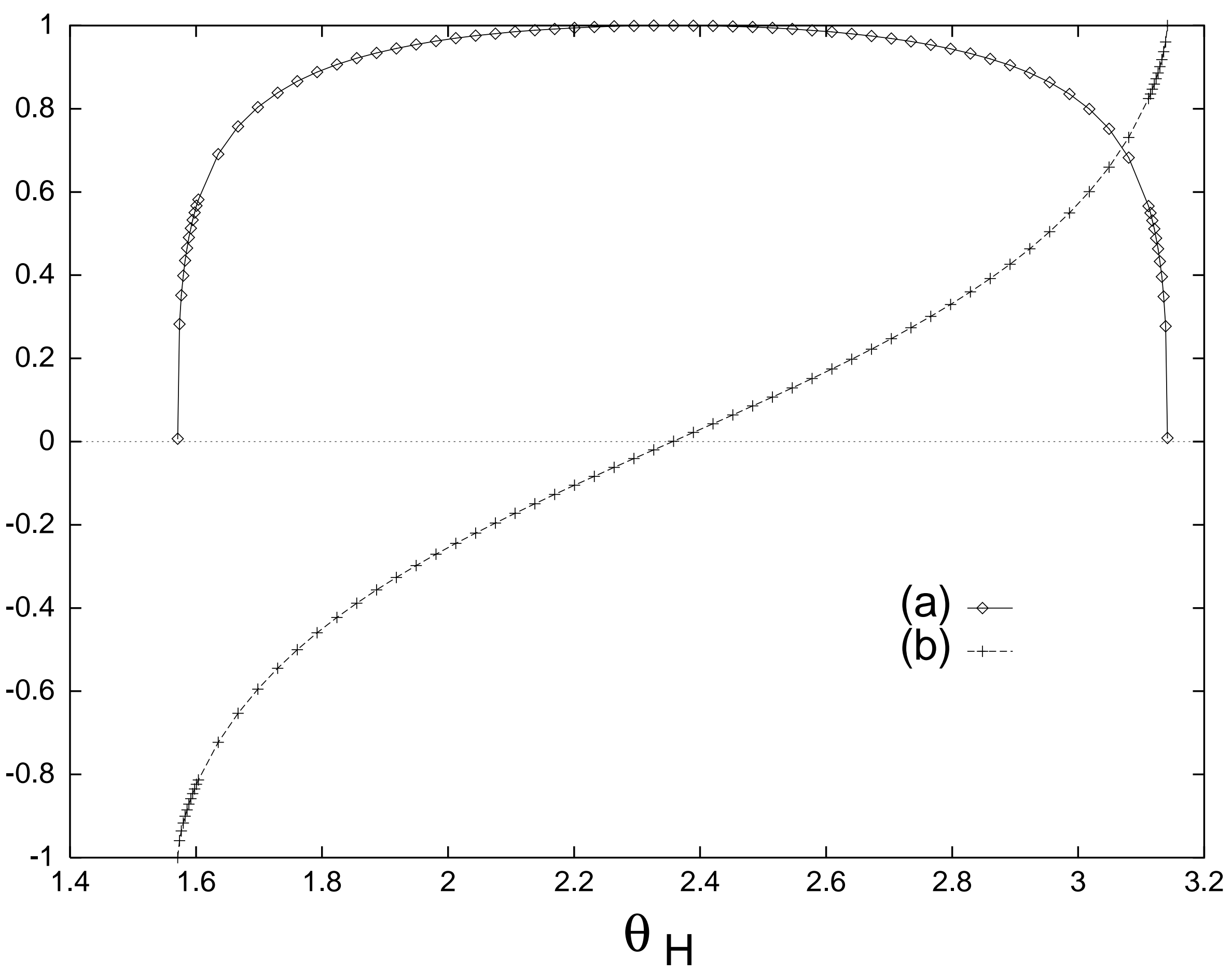




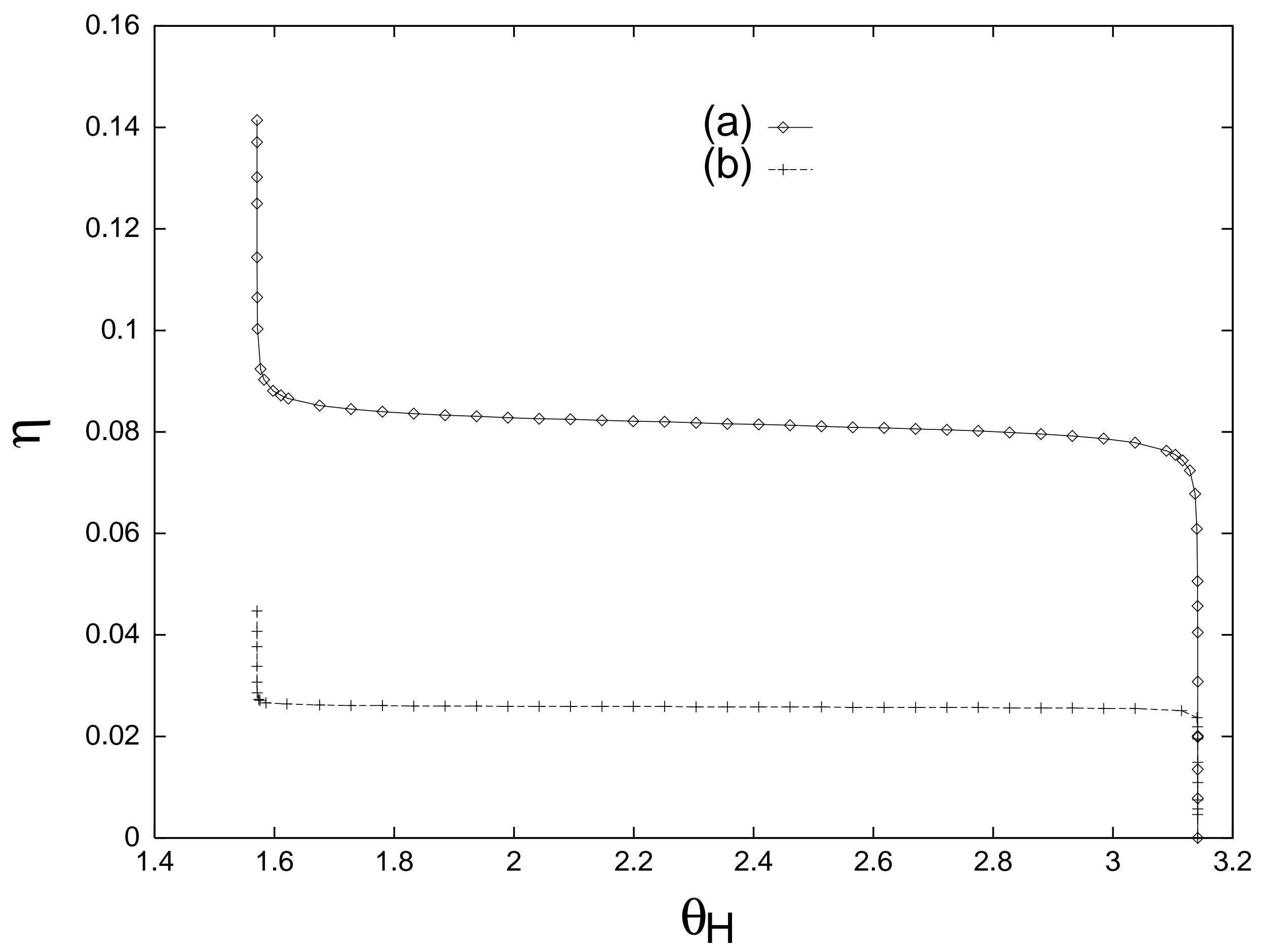




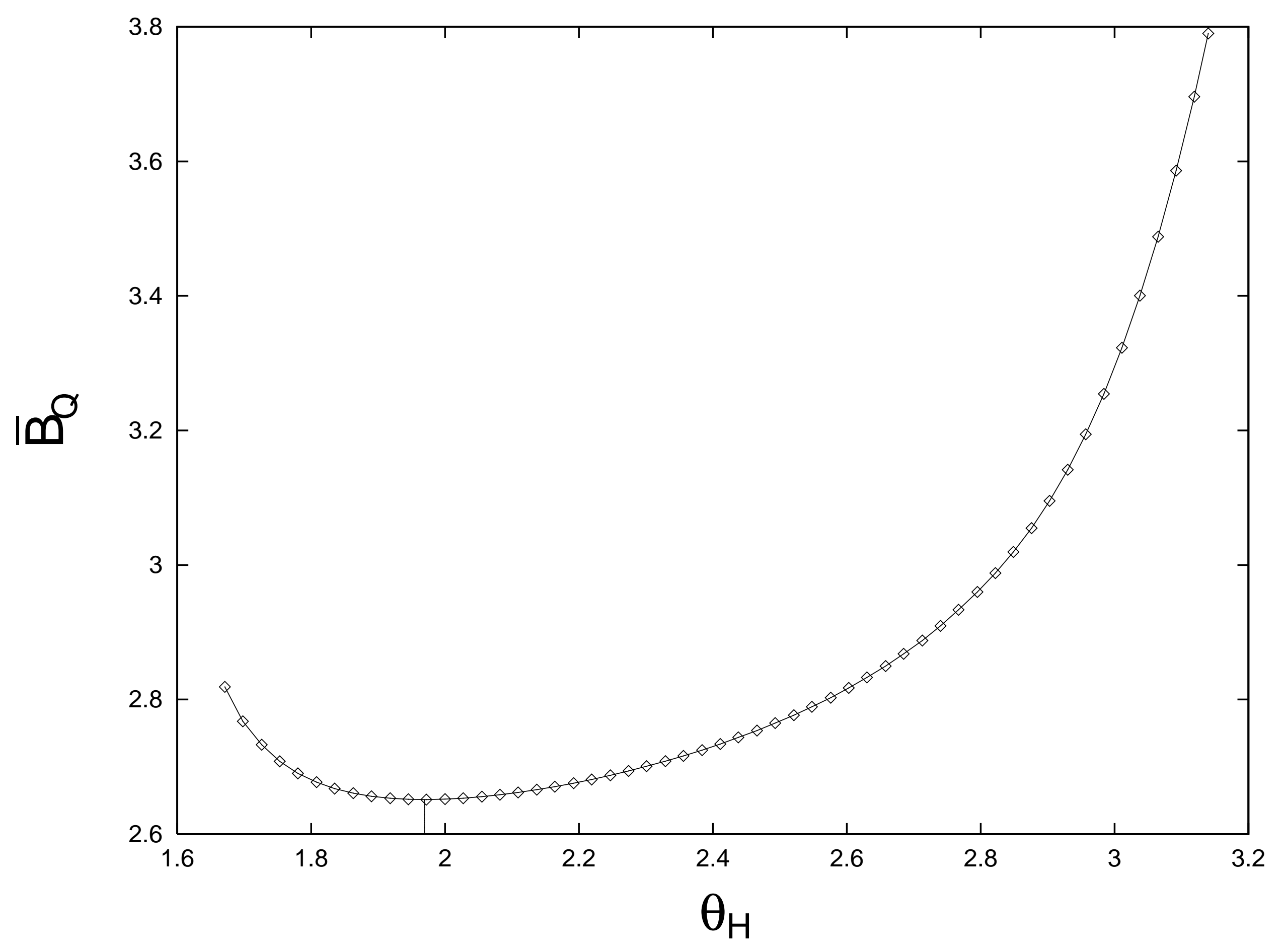

